\title{
Cuidados fisioterapêuticos a mulher em situação de depressão pós-parto: revisão
}

\section{integrativa}

\author{
Physiotherapeutic care to women in situation of postpartum depression: integrative review \\ Atención fisioterapéutica a mujeres en situación de depresión posparto: revisión integradora
}

Recebido: 05/12/2021 | revisado: 10/12/2021 | aceito: 11/12/2021 | publicado: 20/12/2021

\author{
Giovana de Amorim Padilha \\ ORCID: https://orcid.org/0000-0003-3538-9768 \\ Centro Universitário Mário Pontes Jucá, Brasil \\ E-mail: giovanna_amorimp@ hotmail.com \\ Shirley Gabriely Rogério do Nascimento Silva \\ ORCID: https://orcid.org/0000-0001-6991-7990 \\ Centro Universitário Mário Pontes Jucá, Brasil \\ E-mail: shirleygabrielly_11@hotmail.com \\ Ailton Mota do Nascimento Galvão \\ ORCID: https://orcid.org/0000-0002-9268-6921 \\ Centro Universitário Mário Pontes Jucá, Brasil \\ E-mail: ailton_fisioterapeuta@hotmail.com \\ Ahyas Sydcley Santos Alves \\ ORCID: https://orcid.org/0000-0001-7103-8389 \\ Centro Universitário Mário Pontes Jucá, Brasil \\ E-mail: contato@ahyas.com.br
}

\begin{abstract}
Resumo
Esta pesquisa bibliográfica de revisão integrativa objetivou discutir e analisar acerca dos cuidados fisioterapêuticos à mulher em situação de depressão pós-parto. A pesquisa foi realizada de 15 de setembro a 26 de novembro de 2021, nas bases de dados BVS e PubMed, com os descritores "fisioterapia" e "depressão pós-parto" com estratégias de busca. Os dados foram extraídos através de matriz de sínteses e analisados descritivamente e por tipificação. Resultaram-se 7 estratégias de buscas e totalizaram-se 14 artigos elegíveis. Discutiu-se através dos resultados a definição, perspectivas e potencialidade dos cuidados fisioterapêuticos à mulher em situação de depressão pós-parto, e, os contextos, ações, atividades e recursos fisioterapêuticos à mulher em situação de depressão pós-parto. Este estudo integrou a literatura e trouxe a análise e discussão quanto aos cuidados fisioterapêuticos à saúde da mulher em situação de depressão pósparto, apresentando as definições da atuação, contextos, ações, recursos, atividades, potencialidades. Frente ao exposto, conclui-se que a atuação fisioterapêutica nos cuidados à mulher em situação de depressão pós-parto é de fundamental importância para o cuidado nesta situação, com uma gama de possibilidades de atuação essenciais e de contribuição nas perspectivas educativas, preventivas, promotoras e reabilitadoras à esta população. Evidencia-se, por fim, a necessidade da continuação do estudo nesta temática que aborde mais especificidades e interseccionalidade deste cuidado.
\end{abstract}

Palavras-chave: Depressão pós-parto; Especialidade de fisioterapia.

\begin{abstract}
This integrative literature review aimed to discuss and analyze the physical therapy care for women in a situation of postpartum depression. The research was carried out from September 15th to November 26th, 2021, in the BVS and PubMed databases, with the descriptors "physiotherapy" and "postpartum depression" with search strategies. Data were extracted through a synthesis matrix and analyzed descriptively and by typing. Seven search strategies resulted, totaling 14 eligible articles. Through the results, the definition, perspectives and potential of physical therapy care for women in a situation of postpartum depression were discussed, and the contexts, actions, activities and physical therapy resources for women in a situation of postpartum depression were discussed. This study integrated the literature and brought the analysis and discussion about physical therapy care to women's health in a situation of postpartum depression, presenting the definitions of action, contexts, actions, resources, activities, potential. Based on the above, it is concluded that the physiotherapeutic action in the care of women in a situation of postpartum depression is of fundamental importance for the care in this situation, with a range of essential action possibilities and contribution to educational, preventive, promoting perspectives. and rehabilitators to this population. Finally, it highlights the need to continue the study on this topic that addresses more specificities and intersectionality of this care.
\end{abstract}

Keywords: Baby blues; Specialty of physiotherapy. 


\begin{abstract}
Resumen
Esta investigación bibliográfica de revisión integradora tuvo como objetivo discutir y analizar la atención fisioterapéutica a las mujeres en situaciones de depresión posparto. La investigación se realizó del 15 de septiembre al 26 de noviembre de 2021, en las bases de datos BVS y PubMed, con los descriptores "physiotherapy" y "postpartum depression" con estrategias de búsqueda. Los datos se extrajeron mediante una matriz de síntesis y se analizaron de forma descriptiva y por tipificación. Se utilizaron siete estrategias de búsqueda y un total de 14 artículos fueron elegibles. Los resultados discutieron la definición, perspectivas y potencialidad de la atención fisioterapéutica a las mujeres en situación de depresión posparto, y los contextos, acciones, actividades y recursos fisioterapéuticos a las mujeres en situación de depresión posparto. Este estudio integró la literatura y aportó el analisis y la discusión sobre la atención fisioterapéutica a la salud de las mujeres en situación de depresión posparto, presentando las definiciones de actuación, contextos, acciones, recursos, actividades, potencialidades. Por todo lo expuesto, se concluye que la actuación fisioterapéutica en la atención a la mujer en situación de depresión posparto es de fundamental importancia para la atención en esta situación, con un abanico de posibilidades de actuación y contribución esencial en las perspectivas educativa, preventiva, promotora y rehabilitadora a esta población. Se evidencia, en fin, la necesidad de la continuidad del estudio en esta temática que aborda más especificidades e interseccionalidad de este cuidado.
\end{abstract}

Palabras clave: Depresión pós-parto; Especialidad de fisioterapia.

\title{
1. Introdução
}

O parto ou nascimento de uma pessoa caracteriza-se, na adoção de vários procedimentos e tecnologias que objetivam torná-lo mais seguro tanto para a mulher quanto para seu bebê. Segundo a Diretriz Nacional de Assistência ao Parto Normal (2017), a cada ano no Brasil, ocorrem cerca de 3 milhões de partos, o que envolve quase 6 milhões de pessoas, entre as parturientes e os seus bebês.

O nascimento vem influenciando parcela significativa da população de diversas formas ao se considerar os aspectos das famílias e os seus meios sociais, antes ou depois do parto (Ministério Da Saúde, 2017).

O puerpério é uma fase da vida que deve receber um olhar importante, pois pode apresentar complicações nos âmbitos fisiológico, psicomental e sociais (Burti et al., 2016).

Ramos et al. (2018) evidenciam que o parto vem apresentando altas taxas de intervenções e sem serem objetivadas apenas nas situações de urgente necessidade, deixando de considerar diversos aspectos da mulher e seu bebê, como os aspectos emocionais envolvidos neste processo. No parto e no período do puerpério é necessário compreender que as mulheres ao procurarem ajuda além da preocupação sobre a sua saúde e a do seu bebê, estão também em busca de uma compreensão mais ampla e abrangente da sua situação, pois para elas e suas famílias o momento da gravidez e do parto, em particular, é único na vida e carregado de fortes emoções. A experiência vivida por elas neste momento pode deixar marcas indeléveis, positivas ou negativas, para o resto das suas vidas (Ministério Da Saúde, 2017, p. 5).

Neste período é possível o surgimento de mudanças no humor e emoções maternas, de formas leves e/ou muitas vezes graves (Ratti; Dias; Hey, 2020), sendo uma fase de intensas alterações no âmbito social, psicológico e físico da mulher.

Ramos et al. (2018) afirmam que a condição de saúde psiquiátrica mais comum no puerpério é a depressão pós-parto (DPP), e que no cenário atual tornou-se um problema de saúde pública em escala mundial. Esta condição, que atinge, aproximadamente $15 \%$ das mulheres pós-parto em geral, caracteriza-se como um transtorno nos diversos desempenhos da mulher-físico, comportamental, cognitivo e emocional (Gonçalves et al., 2018).

A DPP pode afetar qualquer mulher, independentemente da idade, raça, etnia ou situação econômica e apresenta diversas causas, fatores e sintomatologia (Ratti et al., 2020). Como causas e fatores podemos citar as modificações químicas da gestação, dificuldade de obter o descanso de que precisam, privação constante do sono (Ratti et al., 2020), histórico de sofrimentos ou transtornos mentais, eventos estressantes, apoio social ou financeiro ausente ou insuficiente (Ramos et al., 2018).

O Ministério da Saúde (2019), ainda cita como causas e fatores desejo e aceitação da gravidez, a preocupação com o desenvolvimento do bebê, o abortamento ou morte fetal em gestações anteriores, mudanças na relação com o companheiro(a) e na rotina da casa e do trabalho e existência de doenças crônicas e de riscos gestacionais. 
Como sinais e sintomas da DPP podemos citar tristeza, irritação, alterações do sono e apetite, fadiga, humor depressivo, perda de interesse nas atividades, sentimentos de culpa ou desânimo, perda de concentração, pensamentos suicidas, sentimentos de preocupação, chorar com mais frequência ou sem motivo aparente, raiva ou revolta, sofrimento exacerbado por dores físicas

Ainda podemos citar problemas estomacais, alteração de apetite, isolar-se, problemas para se relacionar ou formar um vínculo emocional ou na capacidade de cuidar do bebê (Ratti et al., 2020), percalços sexuais, redução da autoestima e do cuidado pessoal (Ramos et al, 2018), ansiedade, pensamento obsessivo, rejeição do bebê e/ou riscos de infanticídio (Gonçalves, 2018).

Os profissionais de saúde, neste contexto de cuidado a essa condição devem, além de suas atuações específicas, estarem vigilantes, acolher a gestante, seu companheiro e sua família, e dar o apoio necessário para o esclarecimento de dúvidas, a reorganização da rotina de vida, e o planejamento futuro do pré-natal, parto e puerpério e cuidado do recém-nascido (Ministério Da Saúde, 2019).

Esses profissionais, dentro do contexto relatado, devem ter o papel de contribuir no compartilhamento e enfrentamento das situações (Ministério Da Saúde, 2019). O profissional da saúde necessita estar devidamente preparado para lidar com os aspectos emocionais individuais do gestar, do parir e do puerpério. (Ratti et al., 2020)

$\mathrm{Na}$ atenção ao parto e puerpério, a Fisioterapia vem se destacando e atuando de diversas formas. A ação fisioterapêutica nesta área aborda diversas possibilidades.

As ações vão desde ações de redução das dores do parto e de facilitar a realização do mesmo até os cuidados mais específicos (Vicente et al., 2017) e de ações e atividades educativas (Silva et al., 2019), assim, sendo vista como essencial para melhorar a prestação de serviços maternos.

Segundo Melo et al. (2021) e Burti et al. (2016), a Fisioterapia nesses campos pode contribuir para a assistência à mulher em diversos graus de atuação e em diversas disfunções e sintomatologias físicas e psicomentais, indo do retorno rápido às condições pré-gravídicas a prevenção de problemas futuros e

surge como tratamento eficaz para reduzir potenciais fatores que podem comprometer a mulher nessa fase, iniciando e implementando intervenções desde o puerpério imediato, tratando alterações musculoesqueléticas, disfunções uroginecológicas, dores, corrigindo posturas e promovendo exercícios físicos e cardiorrespiratórios, orientando a amamentação e o retorno da mulher às atividades de vida diária, utilizando de diversos recursos (p.2).

O estudo de Melo et al. (2021) que tratou sobre as intervenções fisioterapêuticas no puerpério imediato relatou que diferentes modalidades de exercícios e recursos nesta condição atuaram na redução de alguns sintomas depressivos.

Portanto, pelo contexto apresentado, faz-se necessária a discussão acerca dos cuidados fisioterapêuticos à mulher em situação de depressão pós-parto e objetivou-se esta discussão na literatura científica através de revisão integrativa.

\section{Metodologia}

Foi realizada uma pesquisa bibliográfica do tipo revisão sistemática integrativa. Adotou-se a abordagem qualitativa de forma descritiva e analítica para guiar esta revisão.

Foram utilizadas as orientações do Relatório de Preferências para Revisões Sistemáticas e Metanálise, o PRISMA-P (Galvão et al., 2015) para sua elaboração. Benachio, Mendonça e Nascimento (2019) definem o estudo bibliográfico, como aquele que vai ser realizado a partir

do registro disponível, decorrente de pesquisas anteriores, em documentos impressos, como livros, artigos, teses etc. Utiliza-se de dados ou de categorias teóricos já trabalhados por outros pesquisadores e devidamente registrados. Os textos tornam-se fontes dos temas a serem pesquisados. O pesquisador trabalha a partir das contribuições dos autores dos estudos analíticos constantes dos textos. 
Esta pesquisa foi realizada do período de 15 de setembro de 2021 a 26 de novembro de 2021. Quanto às etapas seguidas, a pesquisa guiou-se nas normatizações de revisões sistemáticas integrativas (Einstein, 2010). Na base Descritores em Ciências da Saúde (DECS, 2021) foram selecionados os descritores que representaram a problemática da pesquisa: fisioterapia, depressão pós-parto, e como estratégia de busca.

Nas pesquisas nas bases de dados foram utilizados os descritores em português, espanhol e inglês, os operadores booleanos AND, OR e NOT, a estratégia de trucagem e as interfaces dos descritores (no singular, plural, entre aspas). Utilizouse nesta pesquisa fontes de portais e bases de dados eletrônicas, as quais foram: o portal BVS (Biblioteca Virtual em Saúde) e o portal PubMed.

Excluíram-se os resultados das bases de dados que se enquadraram nos critérios de inclusão, mas que eram livros, editoriais e/ou publicações governamentais; estavam duplicados nas bases de dados; e, não estavam disponíveis para download (pagos).

Foram incluídos nesta revisão artigos científicos dos portais e bases eletrônicas: nacionais e/ou internacionais citadas anteriormente; que trouxeram no título, no resumo, no assunto, nas palavras chaves ou texto completo os descritores selecionados para esta revisão e representaram o objetivo e problema da pesquisa; de abordagem qualitativos, quantitativos, quali-quanti e/ou quanti-quali; publicados de 2016 a 2021; de pesquisas bibliográficas e/ou pesquisas empíricas (experimentais/primárias); em português, inglês e/ou espanhol; e em publicações completas.

Os artigos que apresentaram o objetivo da pesquisa e/ou alguma abordagem da temática que gerasse discussão para esta pesquisa após leitura do título, resumo e seu texto na íntegra foram selecionados e elegidos após a aplicação dos critérios de inclusão e exclusão nesta revisão.

Utilizou-se a leitura e fichamentos através de quadros criados especificamente para esta revisão (com a análise crítica e individual de cada artigo) para extração das informações, coletando dados de identificação como título, autores, etc, e, dados conceituais dos artigos, como os principais resultados, procedimento e o que o artigo abordava referente à problemática desta revisão que contribuísse para a mesma.

Os dois revisores realizaram a pesquisa, revisão e análise de forma independente, crítica, comparativa, discursiva e descritiva, buscando a interpretação dos dados de forma transparente em cada etapa através da observação, contagem numérica, descrição e classificação.

Para efetuar o processo de organização e ordenamento das ideias emergidas da leitura e fichamentos, utilizou-se a tipificação/categorização (a priori e posteriori), escolhendo e criando-se as categorias de temas para as discussões.

\section{Resultados}

O Quadro 1, representa a matriz de síntese das informações que foram extraídas dos artigos selecionados nesta revisão. No quadro observa-se informações quanto à identificação e os principais temas e discussões abordados referentes a problemática desta revisão e suas principais contribuições para a pesquisa. 
Quadro 1. Informações de identificação e principais contribuições.

\begin{tabular}{|c|c|c|}
\hline Título do artigo (em português) & Autores e ano de publicação do estudo & $\begin{array}{l}\text { Principais contribuições para a discussão desta } \\
\text { revisão }\end{array}$ \\
\hline 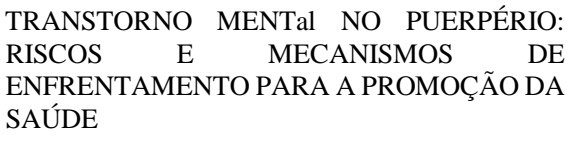 & $\begin{array}{l}\text { Luciana Pessoa Maciel, Jackline Carvalho } \\
\text { Carneiro Costa, Gescianne Mychelle } \text { Benigno } \\
\text { Campos, Nadja Maria Dos Santos, Rosana Alves } \\
\text { De Melo, Lucyo Flávio Bezerra Diniz, } 2019 .\end{array}$ & $\begin{array}{l}\text { Traz cuidados em saúde mental no âmbito da } \\
\text { promoção a saúde que o fisioterapeuta pode ofertar } \\
\text { à mulher em situação de depressão pós-parto. }\end{array}$ \\
\hline $\begin{array}{l}\text { CONSEQUÊNCIAS DA DEPRESSÃO POS- } \\
\text { PARTO NO DESENVOLVIMENTO INFANTIL: } \\
\text { REVISÃO INTEGRATIVA }\end{array}$ & $\begin{array}{l}\text { Rodrigues, Wdyane Layane Da Costa; Branco, } \\
\text { July Grassiely De Oliveira; Facundo, Sue Helem } \\
\text { Bezerra Cavalcante; Costa, Francisca Bertilia } \\
\text { Chaves; Oliveira, Célida Juliana De., } 2019 .\end{array}$ & $\begin{array}{l}\text { Fala das possibilidades de atuação do } \\
\text { fisioterapeuta à mãe em situação de depressão pós- } \\
\text { parto e também da atuação indireta à mãe através } \\
\text { do cuidado ao desenvolvimento do bebê. }\end{array}$ \\
\hline 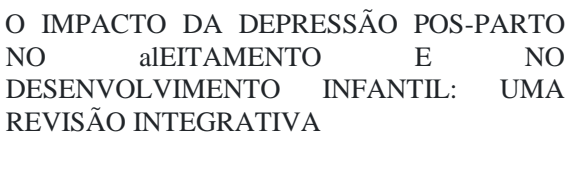 & $\begin{array}{l}\text { Matteussi Lino, C., De Barros Ribeiro, Z., De } \\
\text { Fátima Possobon, R., \& Casati Lodi, J., } 2019 .\end{array}$ & $\begin{array}{l}\text { Fala das possibilidades de atuação do } \\
\text { fisioterapeuta a mãe em situação de depressão pós- } \\
\text { parto em aleitamento e também da atuação indireta } \\
\text { à mãe através do cuidado ao desenvolvimento do } \\
\text { bebê. }\end{array}$ \\
\hline $\begin{array}{l}\text { DEPRESSÃO ENTRE PUÉRPERAS: } \\
\text { PREValÊNCIA E FATORES ASSOCIADOS }\end{array}$ & $\begin{array}{l}\text { Juliana Mano Hartmann, Raul Andrés Mendoza- } \\
\text { Sassi, Juraci Almeida Cesar, } 2017 .\end{array}$ & $\begin{array}{l}\text { Traz reflexões sobre fatores preveníveis à } \\
\text { depressão pós-parto que o fisioterapeuta pode } \\
\text { atuar no âmbito da prevenção. }\end{array}$ \\
\hline $\begin{array}{l}\text { SATISFAÇÃO DE PUÉRPERAS } \\
\text { INTERVENÇÃO APÓS } \\
\text { EDUCAÇÃIO EM SAÚDE }\end{array}$ & $\begin{array}{l}\text { Jordana Barbosa Da Silva, Gabrielle Esther Doi, } \\
\text { Laysse Candido Da Silva, Maria Izabel Feltrin, } \\
\text { Talita Gianello Gnoato Zotz, Raciele Ivandra } \\
\text { Guarda Korelo, Rubneide Barreto Silva Gallo, } \\
2019 \text {. }\end{array}$ & $\begin{array}{l}\text { Traz reflexões sobre intervenções educacionais em } \\
\text { saúde que o fisioterapeuta pode atuar com a mulher } \\
\text { em situação de depressão pós-parto. }\end{array}$ \\
\hline $\begin{array}{l}\text { AUTOEFICÁCIA PARA AMAMENTAÇÃO E } \\
\text { DEPRESSÃO PÓS-PARTO: ESTUDO DE } \\
\text { COORTE }\end{array}$ & $\begin{array}{l}\text { Erika De Sá Vieira, Nathalia Torquato Caldeira, } \\
\text { Daniella Soares Eugênio, Marina Moraes Di } \\
\text { Lucca, Isília Aparecida Silva, } 2018 .\end{array}$ & $\begin{array}{l}\text { Traz reflexões sobre a atuação orientacional do } \\
\text { fisioterapeuta no âmbito da amamentação como } \\
\text { fator de prevenção da depressão pós- parto à mãe.. }\end{array}$ \\
\hline $\begin{array}{l}\text { AÇÕES DO PROGRAMA DE PUERPÉRIO NA } \\
\text { ATENÇÃO PRIMÁRIA: UMA REVISÃO } \\
\text { INTEGRATIVA }\end{array}$ & Tatiane Baratieri, Sonia Natal, 2017. & $\begin{array}{l}\text { Demonstra ações de atenção primária que podem } \\
\text { ser realizadas com a mulher em depressão pós- } \\
\text { parto, inclusive pela Fisioterapia. }\end{array}$ \\
\hline $\begin{array}{l}\text { O PAPEL DA FISIOTERAPIA PÉLVICA NO } \\
\text { PUERPÉRIO IMEDIATO - UMA REVISÃO } \\
\text { SISTEMÁTICA }\end{array}$ & $\begin{array}{l}\text { Erica Feio Carneiro Nunes, Barbara Gonçalves, } \\
\text { Gustavo Fernando Sutter Latorre, } 2019 .\end{array}$ & $\begin{array}{l}\text { Elenca diversas possibilidades de condutas } \\
\text { fisioterapêuticas à mulher nesta situação. }\end{array}$ \\
\hline $\begin{array}{l}\text { OS BENEFÍCIOS DA } \\
\text { PÉLVICA NA MELHORA DA LIBIDA NO } \\
\text { PERÍODO PÓS-PARTO }\end{array}$ & $\begin{array}{l}\text { Carla Nascimento Dos Santos Menezes, Ítalo } \\
\text { Menezes Barbosa, Renata Mírian De Souza Lins, } \\
\text { Maria Glesilene Ponte Peres, } 2021 .\end{array}$ & $\begin{array}{l}\text { Traz algumas possibilidades de condutas } \\
\text { fisioterapêuticas relativas ao libido à mulher nesta } \\
\text { situação. }\end{array}$ \\
\hline $\begin{array}{l}\text { ValIDAÇÃO DE UM MANUal DE CUIDADOS } \\
\text { FISIOTERAPÊUTICOS NO PÓS-PARTO PARA } \\
\text { PUÉRPERAS }\end{array}$ & $\begin{array}{l}\text { Jordana Barbosa Da Silva, Maria Izabel Feltrin, } \\
\text { Laysse Candido Da Silva, Gabrielle Esther Doi, } \\
\text { Talita Gianello Gnoato Zotz, Raciele Ivandra } \\
\text { Guarda Korelo, Rubneide Barreto Silva Gallo, } \\
\text { 2021. }\end{array}$ & $\begin{array}{l}\text { Traz reflexões sobre uma intervenção educacional } \\
\text { em saúde com diversas condutas que o } \\
\text { fisioterapeuta pode atuar com a mulher nesta } \\
\text { situação. }\end{array}$ \\
\hline $\begin{array}{lll}\text { FISIOTERAPIA NO } & \text { CONTROLE } & \text { DAS } \\
\text { EMOÇOEES DURANTE } & \text { O PRÉ-NATal: } & \text { UM } \\
\text { ENSAIO CLÍNICO RANDOMIZADO } & \end{array}$ & $\begin{array}{l}\text { Marina Baia Do Vale, Cibele Nazaré Câmara } \\
\text { Rodrigues. Ana Paula Moreira Sales, Thaynara } \\
\text { Luize Das Mercês, Gissele Maria Figueiredo } \\
\text { Nunes, João Paolo Bilibio, 2021. }\end{array}$ & $\begin{array}{l}\text { Relata atuações da fisioterapia no controle de } \\
\text { emoções da mulher neste período e de ações } \\
\text { preveníveis. }\end{array}$ \\
\hline 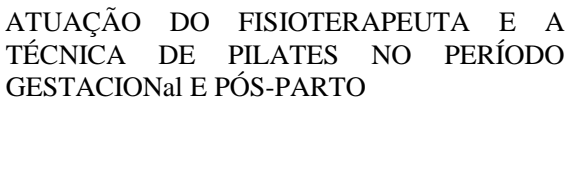 & $\begin{array}{l}\text { Thais Mária Alves De Sousa, Cristina Pereira Da } \\
\text { Silva, Jeandson Ximenes Do Prado, Erianne } \\
\text { Regylla Do Monte Silva, Ana Karina Campelo } \\
\text { Lima Queiroz De Sousa, Anne Karynne Da Silva } \\
\text { Barbosa, 2020. }\end{array}$ & $\begin{array}{l}\text { Traz discussões sobre o uso do pilates que o } \\
\text { fisioterapeuta pode atuar com a mulher nesta } \\
\text { situação. }\end{array}$ \\
\hline $\begin{array}{lcccr}\text { DOR } & \text { E } & \text { FATORES } & \text { ASSOCIADOS } & \text { EM } \\
\text { PUÉRPERAS } & \text { DEPRIMIDAS } & \text { E } & \text { NÃO } \\
\text { DEPRIMIDAS } & & & & \end{array}$ & $\begin{array}{l}\text { Rita Di Cássia De Oliveira Angelo, Lorena } \\
\text { Ferreira Sabino, Paulo Adriano Schwingel, } \\
\text { Antonio Plauto Oliveira Lima, Carla Fonseca } \\
\text { Zambaldi, Amaury Cantilino, Everton Botelho } \\
\text { Sougey, 2016. }\end{array}$ & $\begin{array}{l}\text { Traz informações sobre avaliação e atuação na dor } \\
\text { e outros fatores associados nesta situação. }\end{array}$ \\
\hline $\begin{array}{l}\text { IMPACTO DA DEPRESSÃO PÓS-PARTO NO } \\
\text { alEITAMENTO MATERNO: UMA REVISÃO }\end{array}$ & $\begin{array}{l}\text { Silva, Victória Marques, Gregório, Débora Maria } \\
\text { Alves, Silva, Larissa Matos, Souza, Mariana }\end{array}$ & $\begin{array}{l}\text { Fala das possibilidades de atuação do } \\
\text { fisioterapeuta à mãe em situação de depressão pós- }\end{array}$ \\
\hline
\end{tabular}




\begin{tabular}{|l|l|l}
\hline INTEGRATIVA DE LITERATURA & $\begin{array}{l}\text { Chastel De Liz, Porfirio, Regiane Baptista } \\
\text { Martins, 2021. }\end{array}$ & $\begin{array}{l}\text { parto e também da atuação indireta à mãe através } \\
\text { do cuidado ao desenvolvimento do bebê. }\end{array}$ \\
\hline
\end{tabular}

Fonte: Autores deste artigo (2021).

Resultaram-se 7 estratégias de buscas para as bases de dados com os descritores selecionados. Após a aplicação das estratégias de buscas nas bases de dados, totalizou 113 achados nas duas bases de dados (101 da base BVS, e 12 PubMed).

Dos achados totais, após a aplicação dos critérios de exclusão e inclusão foram selecionados 16 artigos para leitura, e após a leitura na íntegra excluiu-se 2, que não abordavam de forma significativa para pesquisa a temática ou alguma contribuição, totalizando assim uma elegibilidade de 14 artigos.

A Figura 1 representa o fluxograma dos resultados das etapas das buscas nas bases de dados e seleções desta revisão, seguindo as orientações de sistematização PRISMA-P.

Figura 1. Fluxograma de resultados das buscas e seleções.

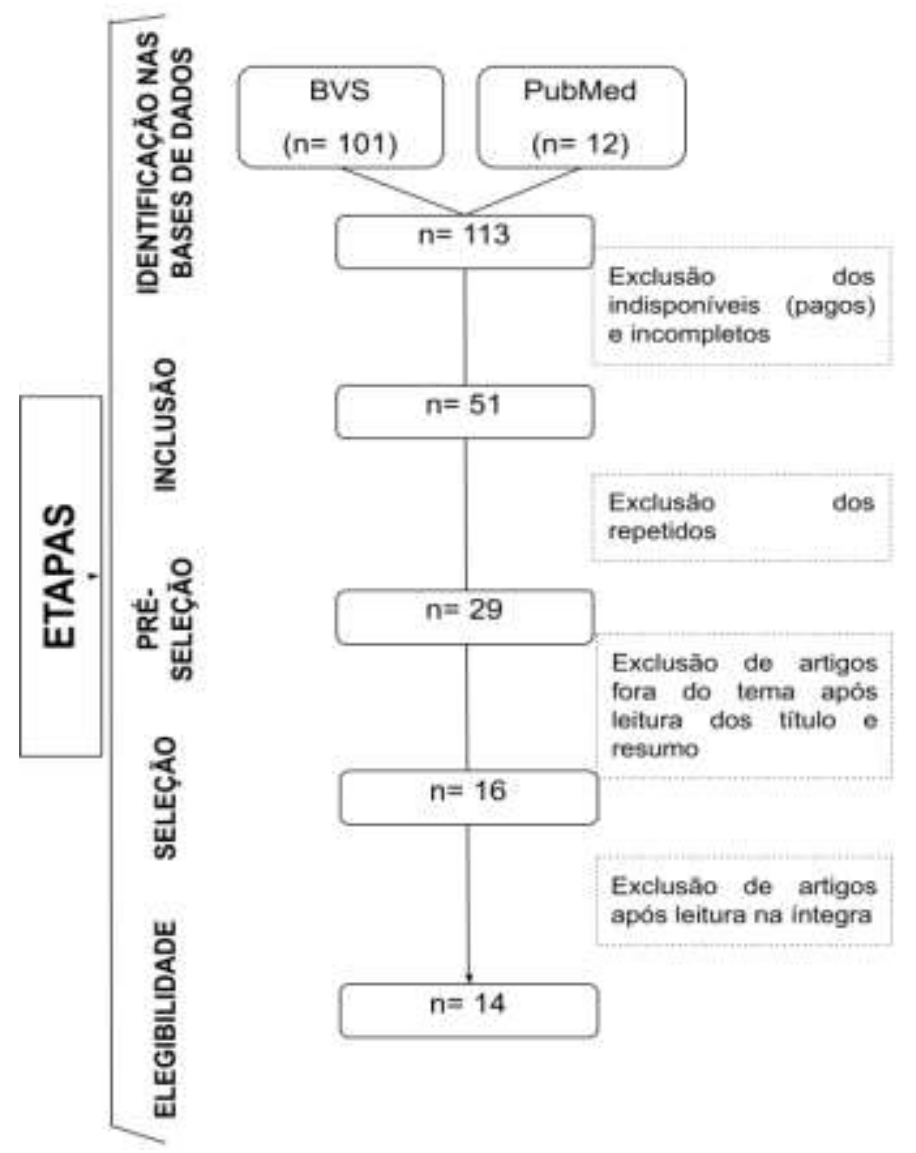

Fonte: Autores deste artigo (2021).

Esta revisão resultou em 2 principais achados (2 categorias para a discussão), após a análise e tipificação pelos dois revisores de forma independente, elencados a seguir e que serão analisados, interpretados e discutidos posteriormente na seção de discussão dos achados:

- Definição, perspectivas e potencialidade dos cuidados fisioterapêuticos à mulher em situação de depressão pósparto; e

- Contextos, ações, atividades e recursos fisioterapêuticos à mulher em situação de depressão pós-parto. 


\section{Discussão}

\subsection{Definição, perspectivas e potencialidade dos cuidados fisioterapêuticos à mulher em situação de depressão pós-parto}

A partir da integração das informações resultantes da literatura desta revisão, podemos inferir que o cuidado fisioterapêutico a mulher em situação de depressão pós-parto é aquele que, de forma integral, da prevenção à reabilitação, busca promover os aspectos de saúde e qualidade de vida à mulher em risco ou já em situação de depressão pós-parto, da gestação até o puerpério remoto, no trabalho individual ou do fisioterapeuta em equipe.

Este cuidado abrange diversos contextos, da avaliação fisioterapêutica até a alta fisioterapêutica, numa visão ampliada do ciclo mãe-bebê mas com foco na mulher e seus determinantes sociais e seus agravos de saúde, com conduta desde a atenção primária até a atenção terciária e diagnóstica.

A atuação fisioterapêutica nos cuidados à saúde da mulher em situação de depressão pós-parto envolve diversas condutas, ações, recursos e técnicas que podem ser aplicadas e desenvolvidas tanto no aspecto educativo, tanto no preventivo quanto no reabilitador, de acordo com a queixa, sintomatologia e situação de saúde demandada pela mulher nesta situação.

Embasando a definição citada acima, iniciamos a discussão dos achados num diálogo com as outras literaturas, apresentando o estudo de Nakamura (2010).

O estudo citado relata que um programa de exercícios fisioterapêuticos pode reduzir o risco de depressão pós parto em novas mães. A pesquisa, em seus procedimentos, recrutou 161 mulheres que recentemente tinham dado à luz.

As participantes foram divididas aleatoriamente em três grupos. O primeiro era composto por 62 delas, que se comprometeram a fazer com seus bebês, uma vez por semana durante dois meses, exercícios físicos orientados por um fisioterapeuta, além de cumprir 30 minutos de aula de educação parental com profissionais da saúde. O segundo, com 73 voluntárias, recebeu apenas o material escrito de educação. O último, com 26, não teve qualquer intervenção. Todas as mulheres foram avaliadas no início do projeto, após oito semanas e, então, quatro meses mais tarde, tiveram que responder questionários sobre bem-estar, depressão e quantidade de exercícios físicos. Segundo os pesquisadores, os resultados indicam que houve melhoras significativas no bem-estar e de sintomas depressivos até o fim das análises no primeiro grupo em comparação com os outros.

O estudo, entre seus diversos achados, também obteve outro resultado importante, este, em relação à prevenção dos sintomas ou da condição de saúde em si.

O número de pacientes identificadas com chance de ter depressão pós-parto foi reduzido em $50 \%$. Já nas que não receberam acompanhamento fisioterapêutico, os índices não se alteraram. Nas mães que receberam só as aulas de educação parental, houve pequena redução dos casos, apenas 5\%, mas nada muito significativo. Para os pesquisadores, isso acontece porque quando mãe e bebê praticam atividades físicas juntos compartilham sentimentos e ativam a área do cérebro responsável pelo afeto e produzem mais serotonina, causando mais prazer na convivência entre eles. Porém, os médicos alertam para a importância da escolha certa de exercícios para não causar efeitos distintos (Nakamura et al., 2010).

\subsection{Ações, atividades e recursos fisioterapêuticos à mulher em situação de depressão pós-parto}

Diversos contextos de atuação fisioterapêutica foram encontrados na literatura desta revisão, os quais, podem fortalecer a gama de cuidados que o fisioterapeuta pode ofertar à essa população na atenção primária (Baratieri e Natal, 2017), secundária e terciária. O Quadro 2, trazido logo abaixo, apresenta a síntese destes contextos. 
Quadro 2 - Contextos de atuação fisioterapêutica nos cuidados à saúde da mulher em situação de depressão pós-parto.

\begin{tabular}{|c|c|c|}
\hline $\begin{array}{c}\text { ATUAÇÃO PREVENTIVA E } \\
\text { ASSISTENCIal NOS TRANSTORNOS E } \\
\text { SOFRIMENTOS MENTAIS }\end{array}$ & $\begin{array}{c}\text { ATUAÇÃO PREVENTIVA NOS } \\
\text { FATORES DE RISCO }\end{array}$ & $\begin{array}{c}\text { ATUAÇÃO NOS FATORES DE } \\
\text { ENFRENTAMENTO }\end{array}$ \\
\hline $\begin{array}{c}\text { ATUAÇÃO NO PLANEJAMENTO } \\
\text { FAMILIAR E GESTACIONal }\end{array}$ & $\begin{array}{c}\text { ATUAÇÃO NO EMPODERAMENTO DE } \\
\text { DIVERSOS ÂMBITOS DA MULHER }\end{array}$ & $\begin{array}{c}\text { ATUAÇÃO EM COMPLICAÇÕES } \\
\text { FÍSICO-FUNCIONAIS }\end{array}$ \\
\hline $\begin{array}{c}\text { ATUAÇÃO NA PROMOÇÃO DA SAÚDE } \\
\text { ATUAÇÃO NAS CONSEQUÊNCIAS NO } \\
\text { alEITAMENTO MATERNO }\end{array}$ & $\begin{array}{c}\text { ATUAÇÃO INDIRetA A MÃE NO } \\
\text { CUIDADO AO DESENVOLVIMENTO } \\
\text { DO BEBÊ }\end{array}$ \\
\hline $\begin{array}{c}\text { ATUAÇÃO EDUCATIVA QUANTO AOS } \\
\text { DIVERSOS AMBITOS DESTE } \\
\text { ADOECIMENTO }\end{array}$ & $\begin{array}{c}\text { ATUAÇÃO NOS FATORES DE } \\
\text { FUNÇÕES SEXUAIS }\end{array}$ & $\begin{array}{c}\text { ATUAÇÃO NO CONTROLE DE } \\
\text { EMOÇÕES }\end{array}$ \\
\hline $\begin{array}{c}\text { ATUAÇÃO NA PROMOÇÃO DE } \\
\text { QUalIDADE DE VIDA E BEM-ESTAR }\end{array}$ & $\begin{array}{c}\text { ATUAÇÃO COM AS TÉCNICAS, } \\
\text { CONDUTAS E EXERCÍCIOS } \\
\text { FISIOTERAPÊUTICOS }\end{array}$ & $\begin{array}{c}\text { ATUAÇÃO NA AValIAÇÃO E } \\
\text { DIAGNOOSTICOS ESPECÍFICOS }\end{array}$ \\
\hline
\end{tabular}

Fonte: Autores deste artigo (2021).

Discute-se nos seguintes parágrafos quanto às ações, recursos e atividades fisioterapêuticos à mulher em situação de depressão pós-parto.

Diversos fatores associados à depressão pós-parto na mulher e cuidados preventivos podem ser campos de atuação do profissional da Fisioterapia.

Alguns desses fatores são citados no estudo de Hartmann et al. (2017) atuação no planejamento familiar e gestacional, suporte em equipe de saúde, acolhimento desde a internação pela, equipe de saúde, atuação em algumas complicações clínica físico-funcionais durante a gravidez, suporte social oferecido pelos profissionais no pré-natal e puerpério, assim como nas que atendem o parto no hospital, materiais educativos Silva et al. (2021) e intervenções fisioterapêuticas de educação em saúde (Silva et al., 2019) para auxiliar na mudança de hábitos e melhorar a qualidade de vida para promoção e prevenção da saúde (Silva et al., 2021), atuação em equipe profissional que se estende ao domicílio.

O estudo de Maciel et al. (2019), que estudou sobre transtornos mentais no puerpério, os riscos e mecanismos de enfrentamento e promoção da saúde à esta mulher trazem que a assistência ofertada por este profissional deve ser de qualidade e com vista a redução de riscos.

Nestes aspectos, o fisioterapeuta deve proporcionar ações, condutas e condições de bem-estar físico, mental e social para assegurar o nascimento de uma criança saudável mas principalmente a promoção da saúde materna, visando a redução da morbimortalidade. $\mathrm{O}$ autor deste estudo relata que essa assistência requer uma visão

holística e humanizada, por meio da construção de um vínculo que transmita confiança e segurança para as puérperas, a fim de evitar desconfortos, minimizar os riscos os quais a mulher se encontra mais vulnerável. Para que isso aconteça, é fundamental um olhar preciso e abrangente, tanto voltado para a promoção da saúde quanto ao processo saúde/doença.

A pesquisa de Vale et al. (2021) reforça essa atuação ao demonstrar em seu estudo que a fisioterapia teve influência positiva no aspecto de controle emocional da gestante que realiza a preparação para o parto e da puérpera com assistência hospitalar e domiciliar fisioterapêutica.

Houve um aumento da confiança, relataram diminuição do medo e ansiedade, e ainda o aprendizado de técnicas que as auxiliaram durante o parto (de posicionamento, controle de dor e respiração) e puerpério, sendo destacadas como 
efetivos os exercícios físicos, a prática de Yoga e os cursos de educação no pré-natal, encorajamento a realização de exercícios físicos.

O fisioterapeuta deve realizar intervenções preventivas, terapêuticas e educativas, buscando a melhoria do nível de conhecimento da mulher desde o seu pré-natal (Maciel et al., 2019).

O empoderamento precoce nessa fase pode evitar possíveis riscos e eventos fisiológicos, fazendo com que o puerpério não seja preenchido por tantas dúvidas e medo do incerto, reduzindo consideravelmente fatores de riscos que conduziram problemas mais graves em sua saúde emocional, não só no processo de prestar assistência do gestar e do parir, mas também um cuidado intenso no período puerperal no qual as atenções estão voltadas quase que exclusivamente para a criança.

Os artigos de Rodrigues et al. (2019), Matteussi et al. (2019), Vieira et al. (2021) e Silva et al. (2021), que abordam a atuação referente ao aleitamento materno e indiretamente à mãe através do desenvolvimento do filho, trazem discussões importantes quanto à atuação fisioterapêutica à esta situação.

O estudo de Rodrigues et al. (2019), traz as seguintes consequências da depressão pós-parto da mulher no desenvolvimento infantil de seu bebê, os quais o fisioterapeuta poderá buscar entender as possibilidade e implantar atuações específicas, melhorando fisicamente e nos aspectos também psicomentais e educativos esse binômio mãe-bebê: interação mãebebê, desenvolvimento emocional e cognitivo da criança que mostra dificuldades de aprendizado, de manter interação social e regular seus estados afetivos, menor comportamento exploratório, percentil de peso baixo, alteração no padrão de sono, problemas de comportamento, interação pobre com suas mães, menos vocalizações, desvio do olhar, sinais de angústia, irritação, choro por um período maior de tempo, comportamento depressivo, regulação de comportamentos hostis.

O estudo de Matteussi et al. (2019) também aborda os cuidados referentes às consequências da depressão pós-parto da mulher no desenvolvimento infantil de seu bebê e também da atuação profissional frente aos impactos no aleitamento materno:

há a necessidade de intervenções ainda durante a gestação, com o intuito de trabalhar a saúde mental e bem-estar materno e, consequentemente, melhorar o desenvolvimento infantil infantil. Dentre alguns dos cuidados que o profissional pode ofertar está o de reduzir a dificuldade ou interrupção na manutenção do aleitamento materno, risco elevado de desmame precoce, a presença de dor e dificuldades físicas do aleitamento materno, a visão exacerbada das dificuldades no manejo da lactação, perda da confiança em seu papel materno e nos benefícios do aleitamento materno materno.

Matteussi et al. (2019) ainda ressalta que a DPP nem sempre é o causada apenas pelo perfil psicológico da mãe mas também, resultante da ausência de rede de apoio. Dessa forma, o fisioterapeuta deve ofertar

uma rede de apoio familiar e profissional, são fundamentais para garantir o diagnóstico adequado, a adesão ao tratamento e o aumento da confiança da mulher frente a sua capacidade de cuidar de seu filho e, consequentemente, amamentar. O apoio profissional contínuo e de boa qualidade para a mãe fortalece o uso das redes de apoio - familiares, grupos de apoio, sociedade -, promove a saúde mental materna e reduz o risco de DPP, além de reduzir as morbidades associadas a este quadro e melhorar a qualidade de vida e vínculo mãe-filho.

O estudo de Vieira et al. (2018), retrata que as mulheres que têm a assistência profissional para a amamentação se sentiram mais seguras e menos ansiosas na função materna, em sua capacidade de interpretar e resolver as dificuldades da amamentação. O estudo evidenciou que

mulheres não deprimidas e com expectativa de amamentar na gestação apresentam menor risco de desenvolver depressão pósparto quando obtêm sucesso no aleitamento materno. Assim, não conseguir amamentar como esperado aumenta o risco de DPP. Outros trabalhos apontam que a presença de sintomas depressivos no pós-parto aumentam as 
chances de dificuldades da díade mãe/bebê na amamentação, diminuem a confiança materna para amamentar, além de elevar a probabilidade de interrupção.

Neste contexto, a mulher passa por algumas disfunções físicas, como por exemplo

deixa de estimular a produção de ocitocina que aconteceria durantes as mamadas e então consequentemente, diminui sua ação benéfica de involução uterina, perda de peso, redução de hemorragia pós-parto e diminuição do risco de cancro de mama e colo de útero. Quando o aleitamento é interrompido, o bebê pode ter mudanças de comportamento repentino como sono irregular e irritação, além de ter um atraso em seu desenvolvimento e até falhas no sistema imune (Silvaet al., 2021).

Visto o exposto, entende-se que é imprescindível que o fisioterapeuta implemente ações para que o bebê tenha um bom desenvolvimento e a relação mãe-filho seja saudável.

Quanto aos aspectos de cuidados físicos-funcionais que o fisioterapeuta pode arcar tanto preventivos quanto reabilitadores à essa mulher, o estudo de Nunes et al. (2019), Angelo et al. (2021) e Menezes et al (2021), trazem algumas situações e condutas: exercícios de contração simples da musculatura do assoalho pélvico (MAP), liberação miofascial local (cervical, lombar, membros superiores e inferiores), correntes terapêuticas analgésicas, trabalho de consciência local, propriocepção, função muscular contrátil, treinamentos básicos de propriocepção e coordenação motora, exercícios livres, função muscular sexual e de libido, cuidados com o risco de prolapso, assoalho pélvico não funcional, massagem abdominal no alívio do desconforto causado pelo acúmulo de gases, prevenção de futuras complicações músculo esqueléticas, alterações posturais, exercícios abdominais combinados com a respiração utilizando os princípios da ginástica Hipopressiva, drenagem linfática manual no combate ao edema, utilização correta e funcional da cinta de contenção abdominal, das modificações do corpo no puerpério, postura correta para amamentar, dormir e banhar o bebê, o treinamento constante da MAP combatendo ou regredindo mais rapidamente a incontinência urinária transitória ou cronificada, uso do pilates (no retorno rápido a condições pré-gravídicas e evitam problemas futuros, redução do trabalho gastrintestinal, má postura, incontinência urinária, diminuição da força abdominal, entre outras).

Por fim, um importante aspecto que a fisioterapia também precisa observar nesta atuação é quanto a avaliação dos aspectos de dor e a intervenção para isso. o estudo de Angelo et al. (2021) demonstrou que, além da relação existente entre as variáveis dor e emoções em puérperas

a intensidade na qual a dor é referida no período pós-parto pode ser um sinal preditivo de depressão. Uma vez que as emoções negativas estão relacionadas à amplificação da percepção dos sintomas físicos, que variam de acordo com os níveis de sofrimento psicológico, essas variáveis podem influenciar na percepção dolorosa. Os achados do presente estudo evidenciam que dor de intensidade moderada a intensa aumenta a probabilidade de a puérpera desenvolver sintomas depressivos.

\section{Considerações Finais}

Este estudo integrou a literatura e trouxe a análise e discussão quanto aos cuidados fisioterapêuticos à saúde da mulher em situação de depressão pós-parto, apresentando as definições da atuação, contextos, ações, recursos, atividades, potencialidades.

Frente ao exposto, conclui-se que a atuação fisioterapêutica nos cuidados à mulher em situação de depressão pós-parto é de fundamental importância para o cuidado nesta situação, com uma gama de possibilidades de atuação essenciais e de contribuição nas perspectivas educativas, preventivas, promotoras e reabilitadoras à esta população.

Evidencia-se, por fim, a necessidade da continuação do estudo nesta temática que aborde mais especificidades e interseccionalidade deste cuidado. 


\section{Referências}

Angelo et al. (2016). Dor e fatores associados em puérperas deprimidas e não deprimidas. Rev. Dor 15 (2).

Baratieri, T, \& Natal, S, (2017). Ações do programa de puerpério na atenção primária: uma revisão integrativa. Ciênc. Saúde Coletiva 24 (11).

Brasil. (2016). Diretriz nacional de assistência ao parto normal. Ministério da saúde, secretaria de ciência, tecnologia e insumos estratégicos.

Brasil. (2017). Diretrizes Nacionais De Assistência Ao Normal Parto. Ministério da Saúde, Brasília.

Brasil. (2019). Saúde da mulher na gestação, parto e puerpério. Nota técnica para organização da rede de atenção à saúde com foco na atenção primária à saúde e na atenção ambulatorial especializada. (2019). Hospital Israelita albert Einstein: Ministério da Saúde, 56 P.: Il.

Burti et al. (2016). Assistência ao Puerpério Imediato: o papel da fisioterapia. Rev Fac Ciênc Méd Sorocaba, 18(4): 193-8.

Descritores Em Ciências Da Saúde. (2021). Decs. *. Ed. Rev. E Ampl. Bireme / Opas / Oms.

Einstein (São Paulo). 2010. Revisão integrativa: o que é e como fazer. Rev Einstein, 8 (1).

Galvão, T. F., Pansani, T. S. A., \& Harrad, D. (2015). Principais Itens Para Relatar Revisões Sistemáticas E Meta-Analises: A Recomendação Prisma. 24 (2).

Gonçalves et al. (2018). Reconhecendo e intervindo na depressão pós-parto. Revista saúde em foco - Edição № 10 .

Hartmann et al. (2017). Depressão entre puérperas: prevalência e fatores associados. Cad. Saúde Pública 33 (9).

Maciel et al. (2019). Transtorno mental no puerpério: riscos e mecanismos de enfrentamento para a promoção da saúde. Rev. Pesqui. Cuid. Fundam. (Online), 11(4): 1096-1102

Matteussi et al. (2019). O Impacto Da Depressão Pos-Parto no aleitamento E No Desenvolvimento Infantil: Uma Revisão Integrativa. Nursing (São Paulo), 23(260), 3506-3510.

Melo et al. (2021). Intervenção fisioterapêutica no puerpério imediato: o que há de evidências na última década? Research, Society And Development, 10(3), E47310312849.

Menezes et al. (2021). Os benefícios da fisioterapia pélvica na melhora da libido no período pós-parto. Revista Cathedral, 3(2), 56-65.

Nascimento, F. De L. S., E. C. C. Benachio, E P. H. De Mendonça. (2019). Procedimentos Metodológicos Empregados Nos Artigos Publicados Na Revista Brasileira Da Educação Profissional E Tecnológica (2008-2017)'. Revista Temas em Educação, 28(1), 60-75.

Nunes et al. (2019). O Papel Da Fisioterapia Pélvica no Puerpério Imediato - Uma Revisão Sistemática. Recima21 - Ciências Exatas E Da Terra, Sociais, Da Saúde, Humanas E Engenharia/Tecnologia. 2(7).

Ramos et al. (2018). Fatores associados à depressão pós-parto: revisão integrativa. Enciclopédia Biosfera, Centro Científico Conhecer. 15(27).

Ratti, G. S., Dias, S., \& Hey, A. P. 2020. Sinais e sintomas da depressão pós parto. Brazilian Journal Of Health Review. 3(5).

Rodrigues et al. (2019). Consequências da depressão pos-parto no desenvolvimento infantil: revisão integrativa. Nursing, 22(250), $2728-2733$.

Silva et al. (2019). Satisfação de puérperas após intervenção fisioterapêutica em educação em saúde. Saúde E Pesquisa, Maringá (Pr). 12(1), 141-150.

Silva et al. (2019). Satisfação de puérperas após intervenção fisioterapêutica em educação em saúde. Saúde e Pesquisa. 12(1) (2019. Artigos Originais - Promoção Da Saúde.

Silva et al. (2021). Impacto Da Depressão Pós-Parto No aleitamento Materno: Uma Revisão Integrativa De Literatura. Revista Científica Multidisciplinar Núcleo do Conhecimento. Ed. 06, 10, 60-74.

Silva et al. (2021). Validação de um manual de cuidados fisioterapêuticos no pós-parto para puérperas. Rev Ciência Em Extensão, 16.

Souza et al. (2020). Atuação do fisioterapeuta e a técnica de pilates no período gestacional e pós-parto. Research, Society And Development, 9(11).

Vale et al. (2021). Fisioterapia no controle das emoções durante o pré-natal: um ensaio clínico randomizado. Research, Society And Development, 10(9).

Vicente, A. C., Lima, A. K. B. S., \& Lima, C. B. (2017). Parto cesárea e parto normal: uma abordagem acerca de riscos e benefícios. Temas em saúde. 17(4).

Vieira et al. (2018). Autoeficácia para amamentação e depressão pós-parto: estudo de coorte. Rev. Latino-Am. Enfermagem; 26:3035. 